\title{
Effect of Humidity on Sterilization Using Photocatalyst
}

\section{Hideharu Shintani*}

Faculty of Science and Engineering, Chuo University, Tokyo, Japan

The photocatalyst equipment consists of a titanium dioxlde $\left(\mathrm{TiO}_{2}\right)$ plate and an ultraviolet lamp. The authors studied if the photocatalyst equipment is practically applicable in sterilizing environmental microorganisms in the health care facility. The number of microorganisms was compared among no sterilization (control), UV alone and the photocatalyst sterilization. As a result, a statistical difference was observed between control and the photocatalyst sterilization against airborne microorganisms $(p<0.01)$ but not against surface microorganisms $(p>0.2)$. In addition, no significant difference between UV alone and photocatalyst $(p>0.2)$, indicating the major function of photocatalyst is not $\mathrm{OH}$ radicals, but $\mathrm{UV}$ exposure, however $\mathrm{OH}$ radicals also contribute when humidity increased because $\mathrm{OH}$ radicals increased when water increased. $\mathrm{OH}$ radicals are from water.

The photocatalyst uses an air sucking system, so it may be ineffective against microorganisms tightly attached to surfaces. However, the effectiveness of the photocatalyst to sterilize airborne microorganisms in the health care facility was successfully confirmed.

Concerning the humidity effect on the photocatalyst sterilization, the authors compared the number of airborne microorganisms in cases of the control, UV alone and photocatalyst sterilization when humidity was changed. A statistical difference was observed between UV alone and the photocatalyst sterilization $(p<0.01)$ when humidity was increased to $60-70 \%$, but not observed between UV and the photocatalyst sterilization $(p>0.2)$ when humidity was around 10$20 \%$. This indicates that maintaining high humidity levels will present satisfactory sterilization results due to a greater production of $\mathrm{OH}$ radicals from exposed $\mathrm{TiO}_{2}$ by UV. Furthermore, no effect of the adsorption of sucked airborne microorganisms on the $\mathrm{TiO}_{2}$ membrane may be speculated.

Nosocomial infection recently collects a great attention in the health care facilities. Nosocomial infections have been reported mostly due to cross-transmission via hands from health care employees to immunodeficient patients. It is confirmed that washing hands frequently is important ways to prevent nosocomial infections in the health care facility. In addition it is indispensable to sterilize airborne and falling microorganisms to diminish nosocomial infections. These airborne and falling microorganisms mostly come from humans. The representative microorganisms related to nosocomial infections have been presented $[1,2]$

According to reports, several sorts of microorganisms and viruses in plates were exposed to the photocatalyst in a model experiment and satisfactory sterilization results were obtained $[1,2]$.The sterilization efficiency of the photocatalyst in the practical use with regard to environmental microorganisms in places such as in the health care facility, however the validation study of the photocatalyst sterilization has not yet been conducted so far. It is keenly required to keep the relatively dirty environment of the health care facility clean to prevent nosocomial infections. Prior to maintaining cleanliness within the health care facility with the use of the photocatalyst, it is necessary to confirm whether the photocatalyst equipment may or may not be practically useful for sterilizing the environmental microorganisms in the health care facility.

In addition the effect of humidity on the photocatalyst sterilization has not been reported, so it must be confirmed the efficiency of the photocatalyst. As one of factor of photocatalyst efficiency, humidity is thought to play an important role with regards to $\mathrm{OH}$ radical production.

The photocatalyst equipment in general consists of an ultraviolet (UV) lamp and an anatase type titanium dioxide $\left(\mathrm{TiO}_{2}\right)$ plate $[1,2]$. With UV irradiation to the $\mathrm{TiO}_{2}$ plate, $0 \mathrm{H}$ radicals are produced $[1,2]$. The produced $\mathrm{OH}$ radicals is of major importance in this sterilization system, The $\mathrm{O}, \mathrm{OOH}, \mathrm{H}$ or $\mathrm{OH}$ radicals (Figure 1) produced from the irradiated $\mathrm{TiO}_{2}$ plate, but the sterilization efficiency of the $\mathrm{OH}$ radicals is superior to other radicals (Figure 1). The role of the $\mathrm{OH}$ radical in sterilization is the proton removal from the microorganisms, which causes the oxidation of the microorganisms and results in successful sterilization. The $\mathrm{OH}$ radical itself changes to $\mathrm{H}_{2} \mathrm{O}$ by the reaction of $\mathrm{OH}^{\cdot}+\mathrm{H}^{+}[1]$.

When the air of the environment contaminated with microorganisms is sucked into the photocatalyst equipment, the microorganisms within the photocatalyst will be sterilized with the combined effect of $\mathrm{UV}$ irradiation and $\mathrm{OH}$ radical exposure produced from the irradiated $\mathrm{TiO}_{2}$. The major UV sterilization mechanism was known as the thymine dimmer, thymine-cytosine, cytosine-cytosine dimer formation (Figure 2), which depends on wavelength. However UV sterilization efficiency with regard to fungi was not satisfactory, especially Aspergillus niger or Rhizopus nigricans, Thus it is speculated that when $\mathrm{UV}$ and $\mathrm{OH}$ radical treatment are combined, the synergistic effect can produce more successful sterilization effects with regard to bacteria and fungi. To confirm this, the authors compared the number of the airborne and falling microorganisms receiving no sterilization, control, UV alone (no $\mathrm{TiO}_{2}$ plate) and photocatalyst $\left(\mathrm{TiO}_{2}+\mathrm{UV}\right)$ sterilization treatment, the results were analyzed using several statistical procedures. The results were as mentioned in advance. $\mathrm{TiO}_{2}$ plate effect was not significant, but humidity effect is significant.

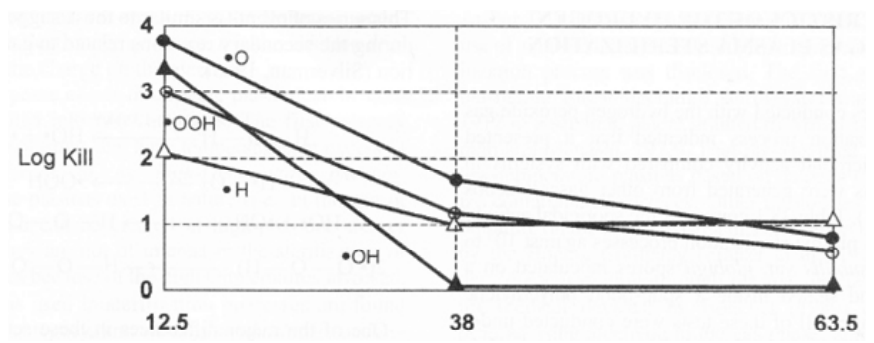

Figure 1: Comparison Efficiency of Sterilization among $\mathrm{OH}, \mathrm{OOH}, \mathrm{H}$ and $\mathrm{O}$ radicals.

*Corresponding author: Hideharu Shintani, Faculty of Science and Engineering Chuo University, 1-13-27, Kasuga, Bunkyo, 112-8551, Tokyo, Japan, Tel: +81425922336, Fax: +81425922336; E-mail: shintani@mail.hinocatv.ne.jp

Received October 08, 2014; Accepted October 09, 2014; Published October 13 , 2014

Citation: Shintani H (2014) Effect of Humidity on Sterilization Using Photocatalyst. Pharmaceut Reg Affairs 3: e141. doi:10.4172/2167-7689.1000e141

Copyright: ( 2014 Shintani H. This is an open-access article distributed under the terms of the Creative Commons Attribution License, which permits unrestricted use, distribution, and reproduction in any medium, provided the original author and source are credited. 


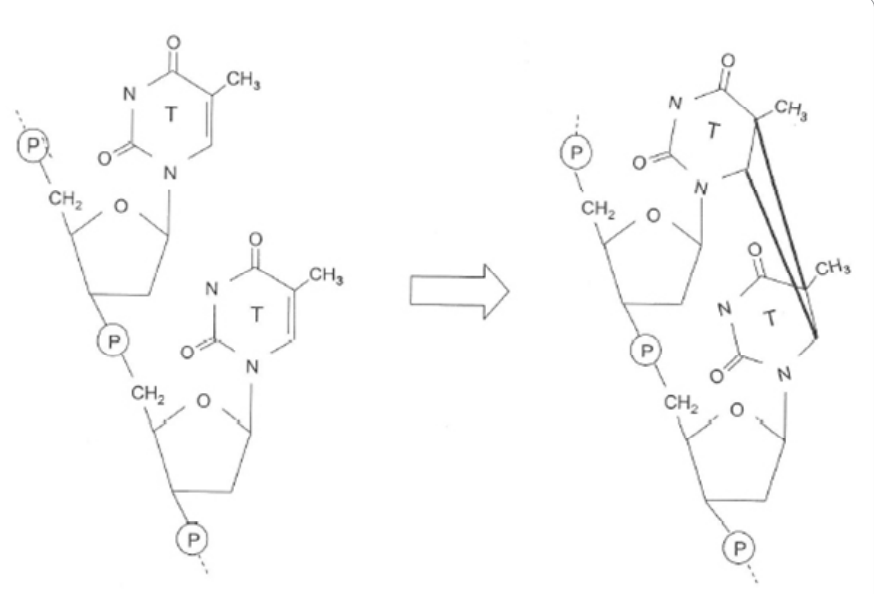

Figure 2: Model Figure of Thymine Dimers by UV Exposure.

It has not yet additionally been confirmed how photocatalyst function in sterilization may change when the humidity around the photocatalyst equipment changes. However, it was speculated that it would be important to maintain relatively high level of humidity
$(60-70 \%)$ to attain satisfactory and reproducible sterilization results with the photocatalyst equipment. That is because the $\mathrm{OH}$ radicals from water (humidity, so it can be speculated that the photocatalyst efficiency might increase when humidity increases. To confirm this, the author studied the sterilization efficiency of the photocatalyst against airborne microorganisms with different levels of humidity around the photocatalyst equipment. As an example, sterilization of ozone gas, peracetic acid gas or dichlorooxide gas requires high humidity such as $90-99 \%$. Hydrogen peroxide does not require such a high humidity, but requires at least more than $70 \%$, indicating Aw (water activity) is an essential factor in sterilization. Photocatalyst sterilization is not exception as it requires at least $60-70 \%$, probably more than $60-70 \%$. It will be clarified by the further experiment.

\section{References}

1. Shintani H, Kurosu S, Miki A, Hayashi F, Kato S (2006) Sterilization Efficiency of the Photocatalyst against Environmental Microorganisms in a Health Care Facility. Biocontyrol Science 11: 17-26.

2. Shintani $H$, Taniai $E$, Miki A, Kurosu S, Hayashi $F$ (2004) Comparison of the Trapping Efficiency of the Airborne microorganisms among air samplers. J Hosp Infect 56: 42-48. 